\title{
Technical Note: Confocal Scanning Laser Microscopy and Quantitative Image Analysis: Application to Cream Cheese Microstructure Investigation
}

\author{
F. Fenoul, ${ }^{*}$ M. Le Denmat, ${ }^{*}$ F. Hamdi, ${ }^{*}$ G. Cuvelier, $\dagger$ and C. Michon ${ }^{1}$ \\ *Groupe Bel, Département Recherche Appliquée 7 boulevard de l'Industrie—BP40077, 41102 Vendôme Cedex, France \\ †AgroParisTech, UMR SCALE no. 1211, ENSIA-INRA-CNAM 1 avenue des Olympiades, 91744 Massy Cedex, France
}

\begin{abstract}
The naked eye observation of cream cheese confocal scanning laser microscopy images only provides qualitative information about its microstructure. Because those products are dense dairy gels, confocal scanning laser microscopy images of 2 different cream cheeses may appear close. Quantitative image analysis is then necessary to compensate for human eye deficiency (e.g., lack of precision, subjectivity). Two kinds of quantitative image analysis were performed in this study: highorder statistical methods and grayscale mathematical morphology. They were applied to study the microstructure of 3 different cream cheeses (same manufacturing process, same dry matter content, but different fat and protein contents). Advantages and drawbacks of both methods are reviewed. The way they may be used to describe cream cheese microstructure is also presented. Key words: cream cheese, microstructure, confocal scanning laser microscopy, image analysis
\end{abstract}

\section{INTRODUCTION}

Because texture is a major quality requirement for cream cheeses, its control is a recurring problem for manufacturers. A better knowledge of the structure of these products would help its optimization. Confocal scanning laser microscopy (CSLM) is a useful technique for studying the microstructure of thick materials. Indeed, it allows the recording of optical sections preventing the out-of-focus radiation, one of the major disadvantages of conventional fluorescence microscopy. Furthermore, sample preparation is soft enough to allow the use of this technique with a wide variety of foods, in particular weak dairy gels because they do not suffer physical disruption.

In the case of cream cheeses, fluorescent labeling of proteins has been developed to observe the gel network

Received July 19, 2007.

Accepted December 5, 2007.

${ }^{1}$ Corresponding author: Camille.Michon@agroparistech.fr
(Herbert, 1998; Llamas et al., 2003). A qualitative analysis of these photographs is sufficient for structure description, but it may not be satisfactory for an objective comparison between 2 pictures. Yet, to ascertain the structural differences responsible for texture variations, it is necessary to objectively quantify the differences between the photographs of different cream cheeses.

A CSLM micrograph, like every computerized image, is basically composed of pixels, which are characterized by their brightness (their gray level value in the case of 8-bit images) and their location (or coordinates). A satisfactory description of this kind of image should then take both into account. For example, first-order statistics (mean, variance, skewness, and kurtosis) provide a good description of the gray level histogram, but they leave the spatial distribution of the pixels out of account. Consequently, they must be completed to get an objective and full description of the image and a useful tool to compare different images.

The concept of image texture refers both to the spatial location and the intensity value of each pixel. Geometric information such as shape or size of objects may be extracted from changes of intensity values from one pixel to another. Many image texture analysis methods have been developed (Bres et al., 2003), and some of them are widely used in the food industry (Zheng et al., 2006). More precisely, high-order statistical approaches such as gray level cooccurrence and pixel-run length matrixes provide numerical features that can be used to statistically compare images and to get global information about their texture. However, through these techniques, no geometric information about objects that may lay in the images can be obtained. Mathematical morphology is another set of image analysis procedures that precisely allow the global characterization of size and shape of those objects (Novales et al., 1998). It can then be used to complete statistical methods.

Those different image analysis techniques have been applied in many fields of the food industry such as beef (Li et al., 1999), expanded food (Gao and Tan, 1996), or potato (Thybo et al., 2004), for example. However, cream cheeses, and more generally dairy gels, did not 
Table 1. Chemical composition of the 3 studied cream cheeses

\begin{tabular}{lcccc}
\hline & $\begin{array}{c}\text { Dry } \\
\text { matter } \\
\text { content } \\
(\%)\end{array}$ & $\begin{array}{c}\text { Fat } \\
\text { content } \\
(\%)\end{array}$ & $\begin{array}{c}\text { Protein } \\
\text { content } \\
(\%)\end{array}$ & $\mathrm{pH}$ \\
cheese & 28.5 & 9.0 & 13.1 & 4.6 \\
\hline A & 28.2 & 12.9 & 10.5 & 4.6 \\
B & 28.4 & 16.5 & 6.9 & 4.7 \\
C & & & & \\
\hline
\end{tabular}

receive particular attention on this specific topic. Herbert et al. (1999) managed to discriminate acid dairy gels from rennet gels through the image analysis of CSLM micrographs, but they focused on milk coagulation, and the composition of the gels achieved is clearly more diluted than manufactured cream cheese.

Thus, the present work aims at developing an image analysis procedure that can be used to get structural information about cream cheese microstructure. The techniques described above (cooccurrence matrixes, run length matrixes, and grayscale mathematical morphology) have been applied to cream cheese CSLM micrographs, and their ability to supply knowledge about the inner microscopic organization of cream cheese is tested.

\section{MATERIALS AND METHODS}

\section{Cream Cheese Selection and Composition Measurements}

The 3 studied cream cheeses were manufactured in the Research and Development Center of the Fromageries Bel in Vendôme, France. Fat and nonfat curds were provided by the Fromageries Bel's plant in Sable sur Sarthe, France. A mixture of those curds, salt, and water was then mixed and heated to $74^{\circ} \mathrm{C}$ with a Stephan UMC5 universal machine (Sympak Group, Lognes, France). The thermostatic control of that universal machine's bowl is ensured by temperature-controlled water. The mixture is then homogenized at a constant pressure of 540 bars with an APV-1000 homogenizing machine (APV Invensys, Evreux, France).

The chemical characteristics (DM, protein, and fat contents, $\mathrm{pH}$ ) of these samples were determined according to the official AOAC methods (AOAC, 2006). Those compositions are given in Table 1 . The DM content is the same for the 3 cream cheeses. On the other hand, they are very different in regard to fat and protein contents.

\section{Confocal Scanning Laser Microscopy}

The fluorescent protein dye Alexa Fluor 546 (Molecular Probes, Leiden, the Netherlands) was used to stain the protein matrix. Fat was not labeled.
A sample of each cream cheese was set down in a cavity slide. The fluorescent labeling was performed following the procedure described in Llamas et al. (2003). The samples were examined on a Leica TCS-DM IRE 2 confocal microscope (Leica GmbH, Heidelberg, Germany), with a $40 \times$ oil immersion objective. The samples were kept at $4^{\circ} \mathrm{C}$ during the observation with a refrigerating cell.

This microscope has a helium/neon laser that was used at an excitation wavelength of $543 \mathrm{~nm}$. The detector monitored fluorescence at wavelengths above 550 $\mathrm{nm}$. For each sample, multiple fields were viewed, and 4 of them were acquired and stored as 8-bit Tiff (format) files. The size of the images was $512 \times 512$ pixels (image width $=187.5 \mu \mathrm{m}$ ), and each pixel was encoded by a number (its gray level) between 0 (black) and 255 (white). Care was taken to avoid the areas near the sides or close to the top of the microscope slides. The 12 images obtained for the 3 cream cheeses are shown in Figure 1.

\section{Image Texture Analysis Methods}

Gray Level Cooccurrence Matrix-Based Features. The cooccurrence matrix (Haralick, 1979) is the estimate of the joint probability $M_{i j}(d, \theta)$ of 2 pixels, a distance $\mathrm{d}$ apart along a given direction $\theta$, having particular gray levels $i$ and $j$. Formally, given an image where GL(x,y) is the gray level of the pixel whose coordinates are $\mathrm{x}$ and $\mathrm{y}$, the cooccurrence matrix $\mathrm{M}(\mathrm{d}, \theta)$ is defined by

$$
\frac{1}{\mathrm{R}} \operatorname{Card}\left\{\begin{array}{c}
M_{i j}(d, \theta)= \\
{\left[\left(x_{1}, y_{1}\right) ;\left(x_{2}, y_{2}\right)\right] /} \\
\left(x_{2}, y_{2}\right)=\left(x_{1}+d \cos \theta, y_{1}+d \sin \theta\right) \\
G L\left(x_{1}, y_{1}\right)=i \\
G L\left(x_{2}, y_{2}\right)=j
\end{array}\right\} ;
$$

$\mathrm{R}$ being a normalization constant defined such as

$$
\sum_{i, j} M_{i j}(d, \theta)=1 .
$$

The dimension of this square matrix is then the numbers of gray level classes (256). $\mu_{x}, \mu_{y}, \sigma_{x}$, and $\sigma_{y}$ are defined as the means and the standard deviations of the row and column sums of the matrix.

Haralick et al. (1973) defined 14 features from this matrix. Unfortunately, these features are not independent, and it is still unclear which feature can be ignored (Zheng et al., 2006). However, the following 5 features are commonly used: 


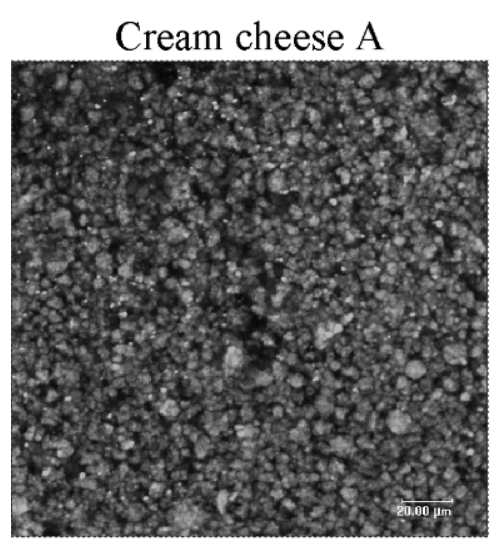

Image A-1

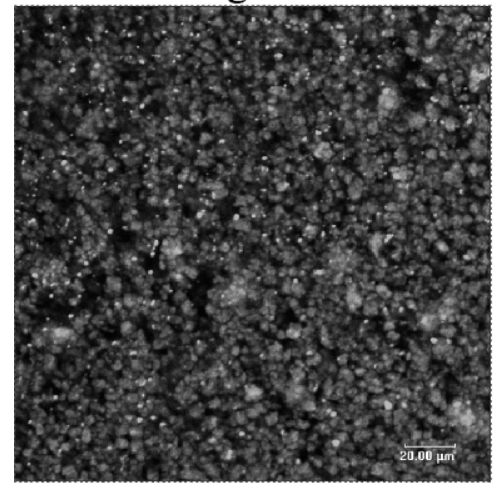

Image A-2

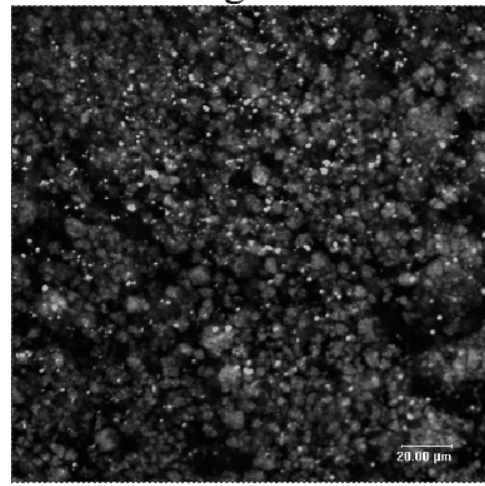

Image A-3

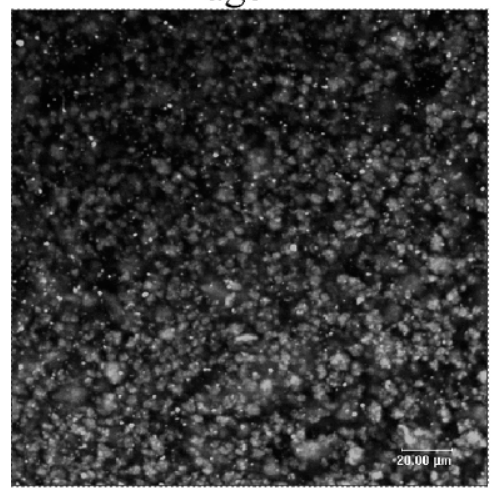

Image A-4

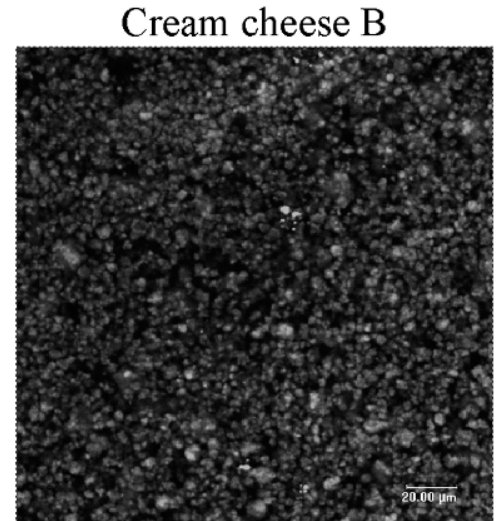

Image B-1

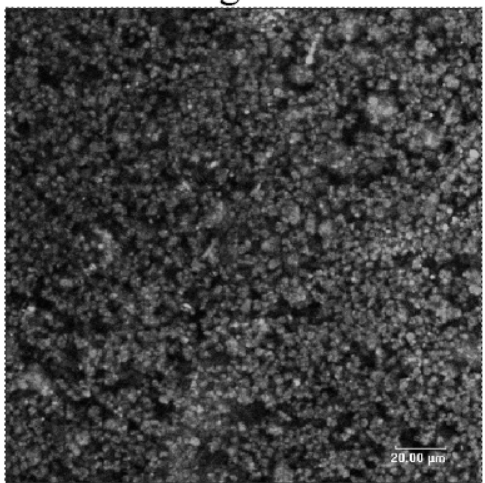

Image B-2

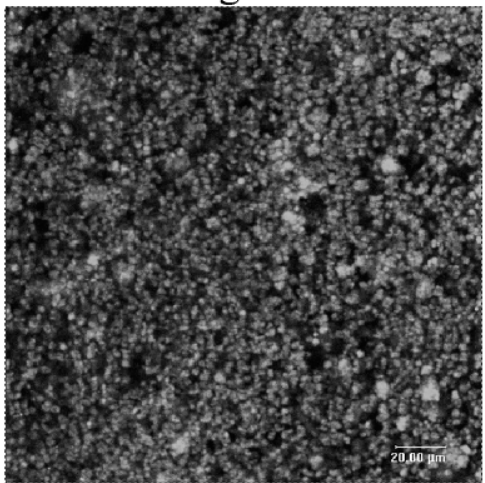

Image B-3

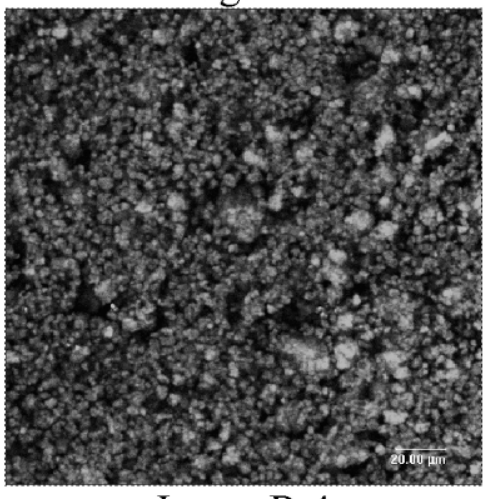

Image B-4
Cream cheese $\mathrm{C}$

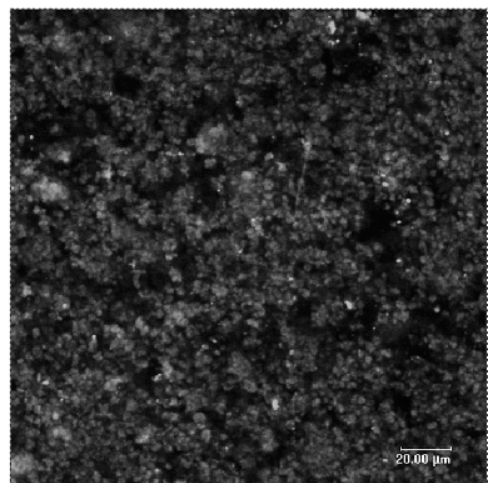

Image C-1

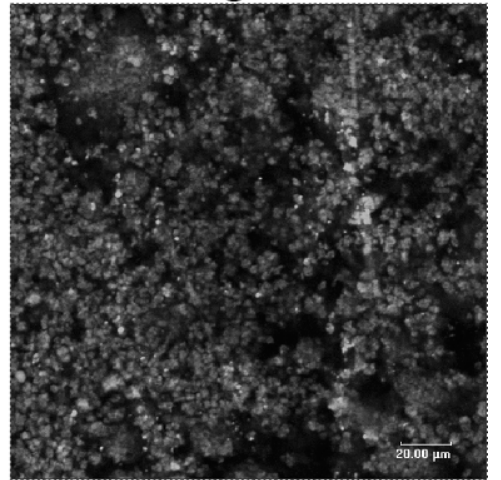

Image $\mathrm{C}-2$

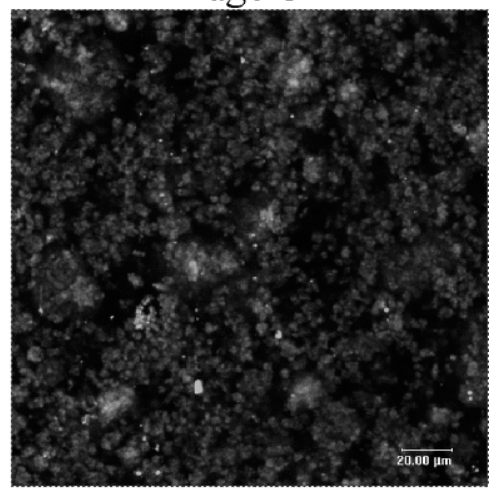

Image C-3

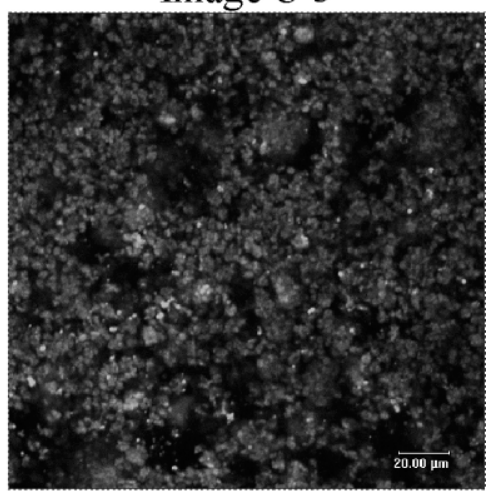

Image C-4

Figure 1. Confocal scanning laser micrographs from 3 different cream cheeses. 
Angular second moment (AngScMom), or energy, shows the uniformity of the image:

$$
\operatorname{AngScMom}(d, \theta)=\sum_{i, j}\left(M_{i j}(d, \theta)\right)^{2} .
$$

Contrast shows the amount of local variations present in the image:

$$
\text { Contrast }(d, \theta)=\sum_{i, j}(i-j)^{2} M_{i j}(d, \theta)
$$

Correlation (Correlat) measures the pixel linear dependencies:

$$
\operatorname{Correlat}(d, \theta)=\sum_{i, j} \frac{i j M_{i j}(d, \theta)-\mu_{x} \mu_{y}}{\sigma_{x} \sigma_{y}} .
$$

Inverse difference moment (InvDifMom), also called local homogeneity, is high when the gray levels of neighboring pixels are similar:

$$
\operatorname{InvDifMom}(d, \theta)=\sum_{i, j} \frac{M_{i j}(d, \theta)}{1+(i-j)^{2}} .
$$

Entropy measures the amount of disorder in the image:

$$
\text { Entropy }(d, \theta)=-\sum_{i, j} M_{i j}(d, \theta) \log M_{i j}(d, \theta) .
$$

Those features are calculated for each cooccurrence matrix $\mathrm{M}(\mathrm{d}, \theta)$. The directions $\theta$ can be selected from 4 different values: $0,45,90$, and $135^{\circ}$. Because there is limited knowledge for selecting the distance $d$, the value of 1 is used (Majumdar and Jayas, 2000). Thus, each image is depicted by 20 features, calculated using the Mazda software (Technical University of Lodz-Institute of Electronics, Lodz, Poland).

Run Length Matrix-Based Features. A pixel run is a sequence of consecutive pixels having the same gray level value. For the direction $\theta$, the number of pixel runs whose gray level is $k$ and whose length is $l$ is stored in a matrix as its $k l$ th entry $Q_{k l(q)}$. This matrix $\mathrm{Q}(\theta)$ is then the so-called run-length matrix for the direction $\theta$. This is not a square matrix: there are indeed 256 possibilities for the gray level and 512 possible run lengths. The size of the run length matrix is then 256 $\times 512$.

Based on this matrix, statistical approaches can be carried out to obtain texture features (Galloway, 1975): Short run emphasis inverse moment (ShrtREmph) indicates the number of short runs:

$$
\text { ShrtREmph }(\theta)=\frac{1}{C} \sum_{k, l} \frac{Q_{k l}(\theta)}{l^{2}} .
$$

Long run emphasis inverse moment (LngREmph) indicates the number of long runs:

$$
\operatorname{LngREmph}(\theta)=\frac{1}{C} \sum_{k, l} l^{2} Q_{k l}(\theta) .
$$

Gray level nonuniformity (GLevNonUni) refers to the gray level distribution:

$$
\text { GLevNonUni }(\theta)=\frac{1}{C} \sum_{k}\left(\sum_{l} Q_{k l}(\theta)\right)^{2} .
$$

Run length nonuniformity (RLNonUni) gives a description of the run length distribution:

$$
\operatorname{RLNonUni}(\theta)=\frac{1}{C} \sum_{l}\left(\sum_{k} Q_{k l}(\theta)\right)^{2} .
$$

Run fraction (Fraction) describes the graininess of the image:

$$
\text { Fraction }(\theta)=\frac{C}{\sum_{k, l} l Q_{k l}(\theta)} .
$$

$\mathrm{C}$ is a normalization constant defined as $C=\sum_{k, l} Q_{k l}(\theta)$.

Thanks to the Mazda Software (Technical University of Lodz-Institute of Electronics, Lodz, Poland), those features are calculated for $\theta=0,45,90$, and $135^{\circ}$. Thus, 20 features depict each image.

Grayscale Mathematical Morphology. Grayscale mathematical morphology is a set of procedures based on the comparison of portions of images to elements of given size and shape called structuring elements (Serra, 1982). Erosions and dilations are the basic transformations from which all the other ones are built. Grayscale erosion consists of replacing the gray level of each pixel of the image by the minimum gray level encountered inside the structuring element when its center is placed on the considered pixel. Erosion causes a reduction of bright objects. On the contrary, dilation causes an increase of bright objects by replacing the gray level of each pixel by the maximum gray level encountered inside the structuring element. Opening is obtained by applying an erosion followed by a dilation. To survive the opening, bright objects have to be larger than the structuring element. In the same way, a closure consists of a dilation followed by an erosion: this operation eliminates dark objects that are smaller than the structuring 


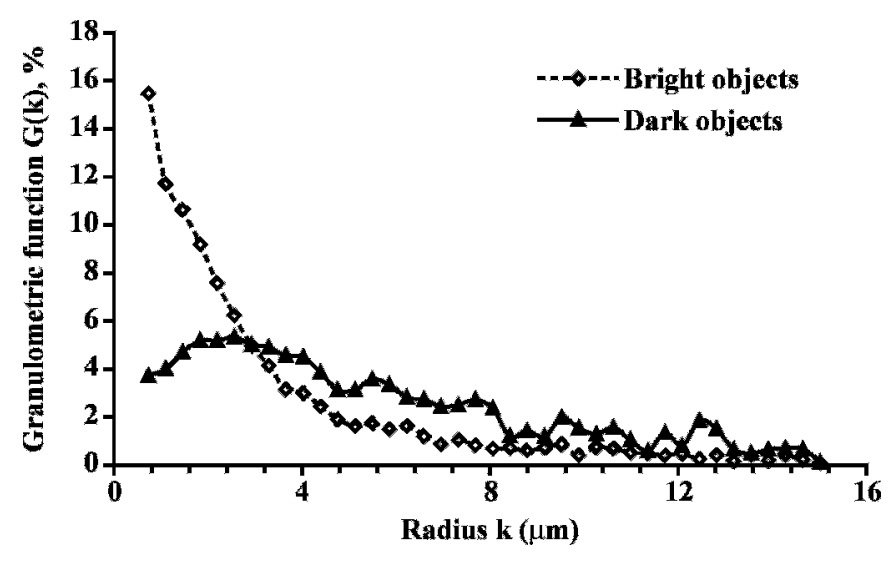

Figure 2. Morphological granulometric curve of image A-4.

element. By applying openings (respectively closures) of increasing size, bright objects (respectively dark areas) progressively disappear. Thus, a granulometric characterization of the image can be derived. The procedure was initially developed for binary images, but it has also been used for determining size distributions from gray level images (Michelland et al., 1989). Forty openings (respectively closures) are applied, with a structuring element radius increasing from $2(4 \times 4$ pixel square $)$ to 41 ( $82 \times 82$ pixel square). GlmOpen ( $k$ ) (respectively GlmClose(k)) is the gray level mean of the image after an opening (respectively a closure) with a $2 \mathrm{k} \times 2 \mathrm{k}$ pixel square structuring element. The morphological granulometric curve $G_{\text {bright }}$ (respectively $G_{d a r k}$ ) of the bright (respectively dark) objects is obtained by deriving GlmOpen(k) (respectively GlmClose(k)). To free those results from the original image gray level mean $G L M_{0}$ and to express them size distribution functions, they are normalized by the initial surface under the curve:

$$
\begin{gathered}
G_{\text {bright }}(k)=\frac{G \operatorname{GmOpen}(k)-\operatorname{GlmOpen}(k+1)}{G L M_{0}-\operatorname{GlmOpen}(41)}, \\
G_{\text {dark }}(k)+\frac{G \operatorname{lm} \operatorname{Close}(k+1)-G \operatorname{GlmClose}(k)}{\operatorname{GlmClose}(41)-G L M_{0}} .
\end{gathered}
$$

Because the image scale is known ( 1 pixel $=0.37$ $\mu \mathrm{m})$, the results can be expressed in micrometers. An example of morphological granulometric curve is given in Figure 2. Those calculations are performed by the Image $J$ software (National Institutes of Health, Bethesda, MD).

\section{Statistical Analysis}

Through the 4 different image analysis methods, 84 features describe each image. Those data are studied by multivariate analysis (principal component analy- sis) and variance analysis (ANOVA), using the JMP 5.1 statistical software (SAS Institute, Cary, NC).

\section{RESULTS}

\section{Cooccurrence and Run Length Matrixes}

Cooccurrence matrix and run-length matrix-based feature means are given in Table 2 for each direction and for the 3 groups of images. Whichever the orientation is chosen for cooccurrence and run length calculations, the differences between the 3 groups of images are similar. For example, cream cheese $\mathrm{C}$ images have greater AngScMom, InvDifMom, and GLevNonUni, but lower contrast than the 2 other groups of images in every direction. That result can be extended to all features. The anisotropic structure of cream cheese is probably responsible for this phenomenon. Consequently, there is no need to study all the features: a single orientation can be chosen.

A principal component analysis was then performed on the features calculated from the $\mathrm{M}\left(1,45^{\circ}\right)$ and $\mathrm{Q}\left(45^{\circ}\right)$ matrices. Ninety-eight percent of the total variation is absorbed by principal components 1 and 2 . Projections of the 12 images on both of them are plotted on Figure 3 . The 3 groups of images are quite well discriminated. Cream cheese $\mathrm{C}$ images appear to be really different from the 2 other groups of images: their coordinates on principal component 1 are clearly lower. However, it seems to be more difficult to discriminate cream cheese A images from cream cheese B images: the latter appears to have coordinates that are slightly higher along principal component 1 and a little bit lower along principal component 2 , but these differences are not statistically significant.

The correlations between the matrix-based features and the principal components are shown in Figure 4. Principal component 1 is well correlated with Entropy, Contrast, and ShrtREmph. On the contrary, its correlation with LngREmph, GLevNonUni, InvDifMom, and AngScMom are close to -1 . Principal component 2 is only significantly correlated with cooccurrence correlation feature (Correlat).

Consequently, it turns out that cream cheese A and $B$ images are quite contrasted and grainy (because of their high entropy and fraction values). Pixel runs are basically short, and the low inverse difference moment values are the expression of the absence of large areas with similar gray values. On the contrary, cream cheese $\mathrm{C}$ exhibits more homogeneous and less contrasted images. Pixel runs are longer (high values of long run emphasis moment), and larger areas with similar gray values can be found in these images. Because these image analysis methods would provide the same results if they were applied to the negatives of these images, it 
Table 2. Gray level cooccurrence and run-length matrixes-based features: means and variance analysis ${ }^{1}$

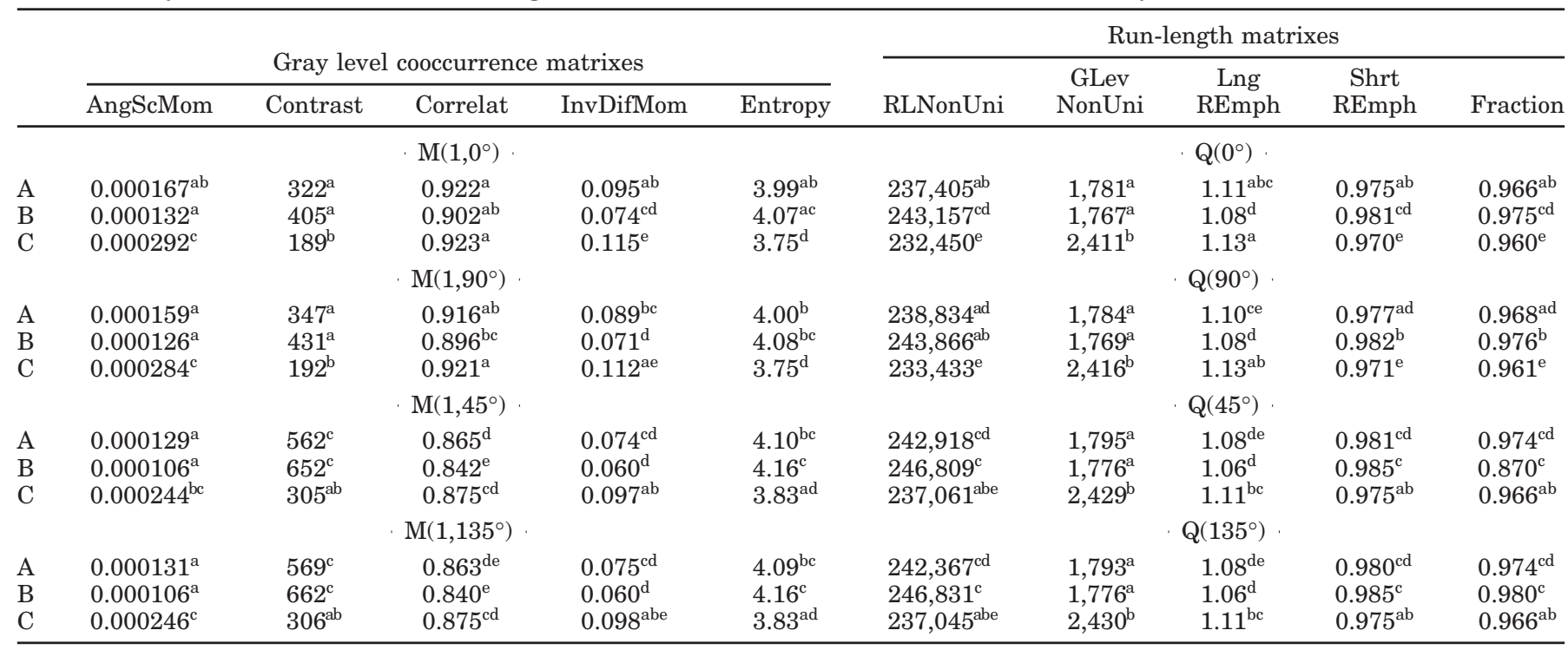

${ }^{\mathrm{a}-\mathrm{e}}$ Different superscripts in a given column and section indicate a statistical significant difference $(P<0.05)$.

${ }^{1}$ AngScMom = angular second moment; Correlat = correlation; InvDifMom = inverse difference moment; RLNonUni = run length nonuniformity; GLevNonUni = gray level nonuniformity; LngREmph = long run emphasis inverse moment; ShrtREmph = short run emphasis inverse moment; Fraction = run fraction.

is not possible to determine through those calculations whether those large areas are dark or bright. That can only be postulated by naked eye observation (in this case it seems that there are large dark areas in cream cheese $\mathrm{C}$ images).

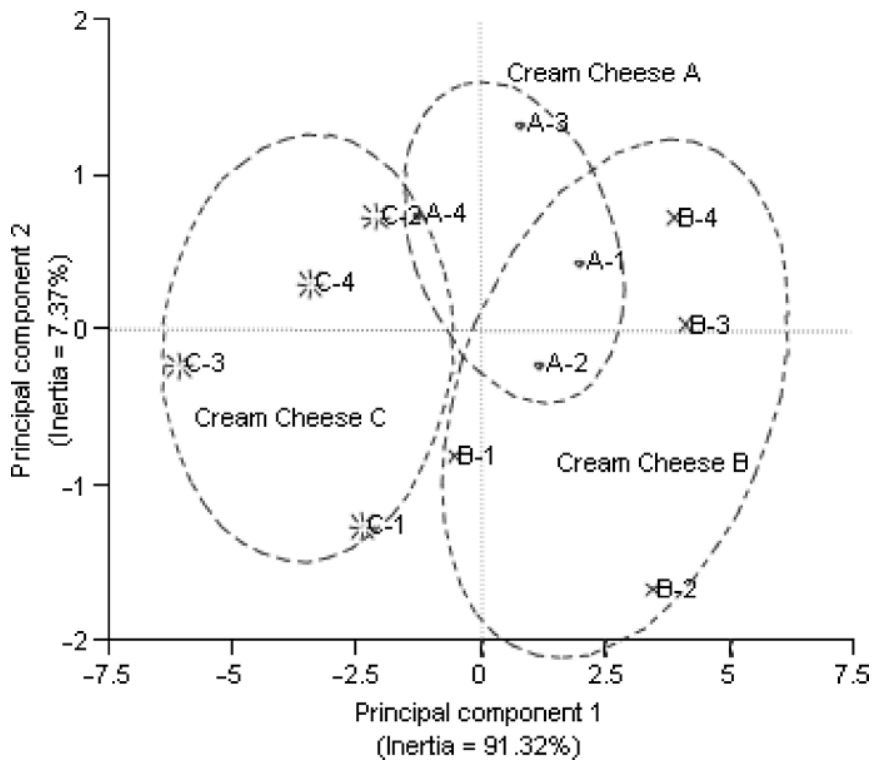

Figure 3. Principal comopnent analysis of the $\mathrm{M}\left(1,45^{\circ}\right)$ and $\mathrm{Q}\left(45^{\circ}\right)$ based features showing the distribution of the 12 images.

\section{Grayscale Mathematical Morphology}

From a qualitative point of view, cream cheeses have a particulate microstructure, built from aggregated clusters. Thanks to grayscale mathematical morphology, it is possible to get knowledge about both the cluster size (which appear as bright objects) and the pore size (which can be considered as dark objects in the

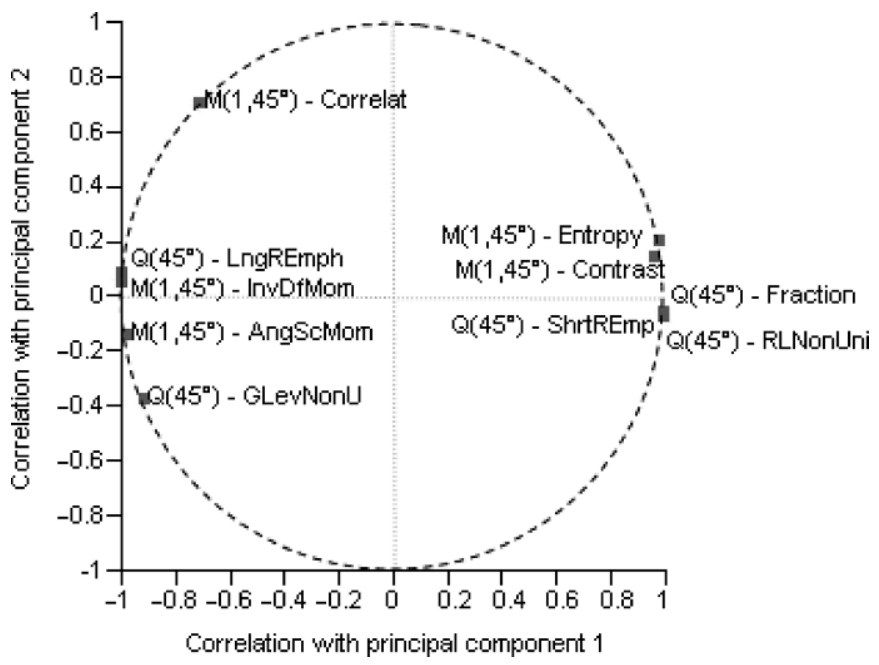

Figure 4. Correlation coefficients between matrix-based features and principal components 1 and 2 . 
a) Bright objects (protein clusters)

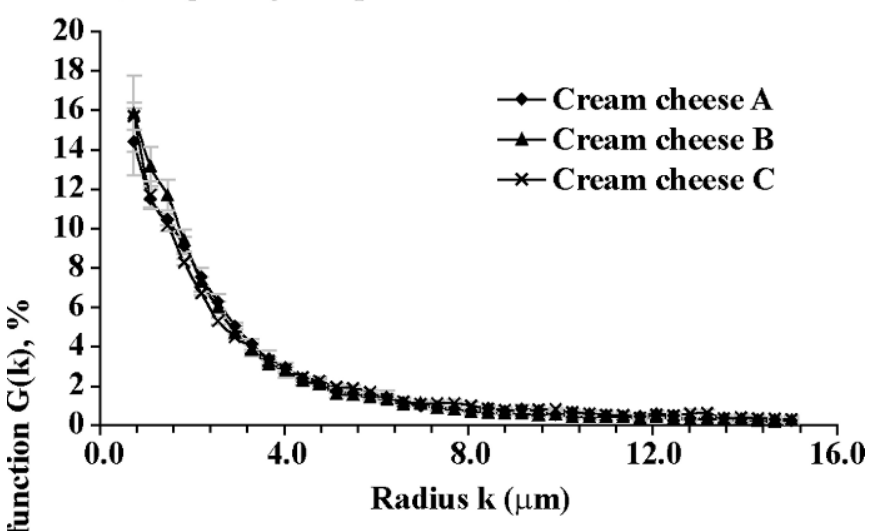

b) Dark objects (pores)

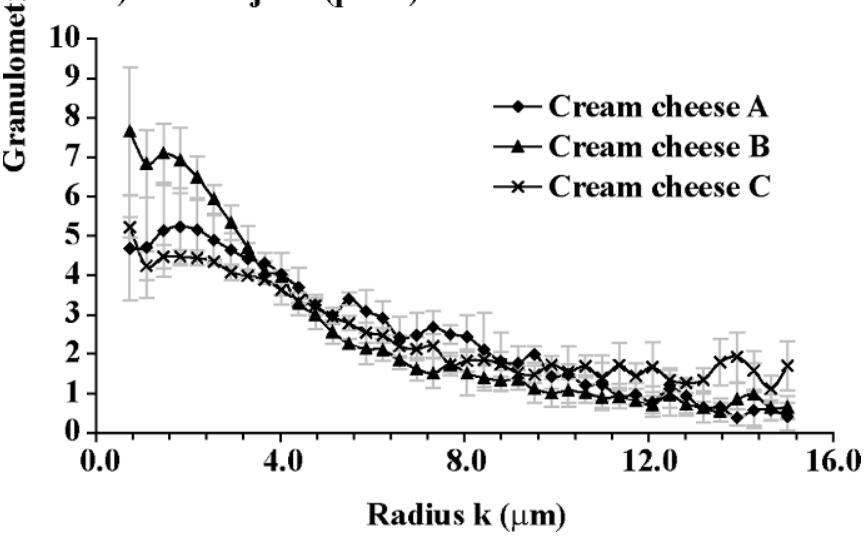

Figure 5. Average granulometric curves.

images). Average granulometric curves are calculated for each group of images and are shown in Figure 5.

When considering the bright object size distributions, it appears that cream cheeses A, B, and C do not exhibit great differences. The 3 of them are built by the aggregation of very small particles: the average radius of bright objects is around $3.3 \mu \mathrm{m}$, but $70 \%$ of them have a radius less than this value (the modal value is between 1.8 and $2.2 \mu \mathrm{m}$ ). Clusters with higher radius than $6 \mu \mathrm{m}$ represent only $15 \%$ of the total surface. As after a $82 \times 82$ pixel square opening, the gray level mean is very low (around 15) for all the images. We can then assume that they contain very few continuous bright objects whose radius is greater than $15 \mu \mathrm{m}$.

In spite of those great similarities, 2 significant differences can be noted among the 3 considered cream cheeses. There are more clusters whose size is included between 0.7 and $1.5 \mu \mathrm{m}$ in cream cheese B than in cream cheeses $\mathrm{A}$ and $\mathrm{C}$. In the same way, cream cheese $\mathrm{C}$ contained fewer clusters whose size is between 1.5 and $2.6 \mu \mathrm{m}$. However, even if those differences are statistically significant $(P<0.05)$, they are still very thin.
With regard to dark objects (pores), it is easier to distinguish the 3 groups of images. Cream cheese B exhibits a very distinctive granulometric profile: in this product, small pores (i.e., with a radius less than 2.9 $\mu \mathrm{m}$ ) represent around $46 \%$ of the total number of pores, whereas their proportion is not over $35 \%$ in cream cheeses $\mathrm{A}$ and $\mathrm{C}$ : it appears that pores and gel particles have almost the same size in cream cheese B. On the contrary, large pores (whose radius is greater than 12 $\mu \mathrm{m}$ ) are cream cheese $\mathrm{C}$ specificity: the amount of such pores is significantly greater in its images than in cream cheeses A and B images (13.7\% vs. less than 6.6\%). Furthermore, as after a $82 \times 82$ pixel square closure, the gray level mean of cream cheese A and B images is quite high (above 225), it can be assumed that there are probably no pores whose radius is greater than 15 $\mu \mathrm{m}$. This result cannot be extended to cream cheese $\mathrm{C}$ images: after the same morphological transformation their gray level mean is low (around 190), and the presence of very large pores may explain this result.

With regard to its pore size distribution, cream cheese $\mathrm{A}$ is intermediate between cream cheeses B and C: it has less small pores than cream cheese $B$ and less large pores than cream cheese $\mathrm{C}$. This result can be compared with the location of cream cheese A images on the principal component analysis made from cooccurrence and run-length matrix features (Figure 3).

\section{Cream Cheese Microstructure}

When considering both grayscale morphology and high-order statistical methods, a portrait of the microstructure of the 3 cream cheeses can be drawn up. As previously mentioned, cream cheeses are particulate gels made from aggregated clusters. The respective roles of fat and of proteins within those clusters are not determined, but it appears that those clusters' size does not depend on the cream cheese composition. Indeed, no significant difference has been calculated in clusters size of the 3 cream cheeses, whereas the protein content varies from 6.9 to $13.1 \%$ and the fat content from 9.0 to $16.5 \%$. It can be assumed that this cluster size is more probably determined by process conditions.

However, it appears that the distance between those clusters, which can be considered as pore size, may be very different from a cream cheese to another. Cream cheese B exhibits very small pores. At the same time, its structure is very grainy: its images are quite contrasted, and this can be attributed to the quick alternation of bright and dark pixels. Moreover, their correlation value is low, which can be interpreted as a lack of continuous substructures. Those results suggest that cream cheese B microstructure is very chiseled (i.e., made from small aggregates that do not make up larger 
clusters). This structure could be compared with a very concentrated suspension of small fat and protein aggregates that are juxtaposed but not entangled. A weaker rheological behavior can be expected for this cream cheese (this point was confirmed experimentally, results not shown).

On the contrary, cream cheese $\mathrm{C}$ exhibits large pores inside its microstructure. This implies that its images have low contrast and entropy values because of the large areas with similar gray levels. In the same way, the short and long run emphasis moments are respectively low and high because of those large dark areas. There is no evidence that a more entangled structure is responsible for the higher correlation values. In fact, because of those large and continuous dark zones, the numerical features cannot ascertain any conclusion about the gel particle structure.

Both mathematical morphology and high order statistical techniques indicate that cream cheese A structure is intermediate between the 2 other products, but its composition is clearly not. The intermediate position of cream cheese A could be considered as an optimum between the opposed effects of fat and protein contents on cream cheese structure. But we could also consider that the final product's structure does not depend on cream cheese composition and that the differences we observed are caused by small process variations.

\section{DISCUSSION}

High-order statistical methods (gray level cooccurrence and pixel run-length matrixes) are powerful tools to compare or to sort out sets of images. They are quite simple to use and they require little statistical data analysis: principal component analysis and variance analysis are enough to classify images that look alike. Moreover, because the probability to differentiate $2 \mathrm{im}$ age texture patterns $\mathrm{A}$ and $\mathrm{B}$ increases if there are more features extracted from the images, their efficiency could be enhanced by calculating all the image texture features. Haralick et al. (1973) defined from the gray level cooccurrence matrixes and by working out all those features for other distance/orientation pairs. However, by increasing the number of parameters, the structural interpretation of the results becomes more difficult. In the case of cream cheeses, anisotropy of the microstructure was checked and leads us to take only one orientation into account. Furthermore, we made the choice to calculate only 5 cooccurrence features. Even so, we showed that structural interpretation of the results is not obvious. Some concepts such as image homogeneity, entropy, or contrast are not easy to visualize, especially when all the images give the same global visual impression, as is the case with cream cheese micrographs. Moreover, those features are highly dependent on image acquisition conditions: better resolution can improve the image contrast, for example. Nevertheless, some parameters are obviously correlated to structural characteristics. As the run length matrix method merges adjacent pixels, the long run emphasis inverse moment (which is highly correlated to cooccurrence inverse difference moment) can reveal the presence (or the absence) of large coherent areas (i.e., large pixel areas with similar gray level values) such as pores or dense protein clusters. Cooccurrence correlation can also be interpreted as an entanglement indicator.

Grayscale morphology is then a useful tool to complete those data. As openings and closures need quite a long calculation time, especially for large structuring elements, this image analysis technique is probably not the best one to use as a routine procedure to sort out a large set of images. Moreover, this technique is highly dependent on the reproducibility of images; as a consequence, the image acquisition procedure has to be as repeatable as possible to get a batch of similar images for each cream cheese.

However, the technique provides quantitative physical data about cream cheese microstructure, such as granulometric profiles. When considering the bright objects (i.e., aggregated clusters), grayscale morphology allows the determination of the size of the elementary particle the gel is built from. Unfortunately, it cannot be used to get the size of the superaggregates those elementary particles form. We have verified that this knowledge cannot be reached by decreasing the number of gray levels from 256 to 64 , for example.

When regarding dark zones; that is, void spaces that lay between the gel particles, granulometric profiles can be studied to get knowledge about the porosity of the gel. No absolute quantitative data can be obtained: the granulometric curves only supply percentages of the total surface occupied by the pores. The fraction of small and large pores is then the only way to compare 2 images. However, even if only relative porosity quantification is considered, some significant differences can be found from a cream cheese to another. Pore granulometric profiles can thus be used to discriminate different cream cheeses. They also may be used to explain rheological behavior differences: a very thin pore granulometric profile can be explained by a very chiseled structure, with small pores spread all over a structure made of juxtaposed gel fragments, and whose rheological strength is poor. On the contrary, the presence of large pores implies the existence of gel strands (made of entangled clusters) to delimit them and, so, may indicate a stronger structure.

As the main aim of this study was to be able to distinguish cream cheeses whose microstructures are not ob- 
viously different, the links between microstructure and rheological behavior have not been formally established. To confirm the hypotheses mentioned above, a similar study should be carried out with cream cheeses that would have been especially selected for their very different rheological characteristics. For example, cream cheeses with very different DM contents would very probably have different rheological characteristics that may be linked to microstructure differences.

\section{CONCLUSIONS}

Grayscale morphology and high-order statistical methods (cooccurrence and run-length matrixes) are both useful tools to investigate cream cheese microstructure. Thanks to them, we showed that in the case of cream cheese, the links between the microstructure and the composition are not obvious. The same kind of study could be operated by labeling fat (e.g., to understand the respective roles of fat and of the proteins within the aggregated clusters cream cheese are made of).

\section{ACKNOWLEDGMENTS}

We sincerely acknowledge Marie Françoise Devaux (INRA, Nantes, France) for the scientific and technical discussions we had before launching this study.

\section{REFERENCES}

AOAC. 2006. Official Methods of Analysis. 18th ed. AOAC International, Gaithersburg, MD.
Bres, S., J. M. Jolion, and F. Lebourgeois. 2003. Traitement et analyse des images numériques. Hermes Science Publications, Paris, France.

Galloway, M. 1975. Texture analysis using grey level run lengths. Comp. Graph. Image Proc. 4:171-179.

Gao, X., and J. Tan. 1996. Analysis of expanded-food texture by image processing. Part I: Geometric properties. J. Food Process Eng. 19:425-444.

Haralick, R. M. 1979. Statistical and structural approaches to texture. Proc. IEEE 67:786-804.

Haralick, R. M., K. Shanmugam, and I. Dinstein. 1973. Textural features for image classification. IEEE Trans. Syst. Man Cybern. 6:610-621.

Herbert, S. 1998. Molecular and microscopic structural characterisation of soft cheeses Multivariate analysis of structural data in relation to texture. PhD Thesis. University of Nantes, France.

Herbert, S. B. Bouchet, A. Riaublanc, E. Dufour, and D. J. Gallant. 1999. Multiple fluorescence labelling of proteins, lipids and whey in dairy products using confocal microscopy. Lait 79:567-575.

Li, J., J. Tan, F. A. Martz, and H. Heymann. 1999. Image texture features as indicators of beef tenderness. Meat Sci. 53:17-22.

Llamas, G., B. Metro, S. Atlan, and A. Riaublanc. 2003. Influence of fat interface on structures obtained by rennet coagulation of emulsions. 3rd NIZO Dairy conference (poster session). Papendal, the Netherlands.

Majumdar, S., and D. S. Jayas. 2000. Classification of cereal grains using machine vision III. Texture models. Trans. ASAE 43:1681-1687.

Michelland, S., B. Schiborr, M. Coster, B. L. Mordike, and J. L. Chermant. 1989. Size distribution of granular material from unthresholded images. J. Microsc. 156:303-311.

Novales, B., S. Guillaume, M. F. Devaux, and M. Chaurand. 1998. Particle size characterisation of in-flow milling products by video image analysis using global features. J. Sci. Food Agric. 78:187-195

Serra, J. 1982. Image analysis and mathematical morphology. Academic Press, London, UK.

Thybo, A. K., P. M. Szczypinski, A. H. Karlsson, S. Dønstrup, H. S. Stødkilde-Jørgensen, and H. J. Andersen. 2004. Prediction of sensory texture quality attributes of cooked potatoes by NMRimaging (MRI) of raw potatoes in combination with different image analysis methods. J. Food Eng. 61:91-100.

Zheng, C., D. W. Sun, and L. Zheng. 2006. Recent applications of image texture for evaluation of food qualities-A review. Trends Food Sci. Technol. 17:113-128. 